OPEN ACCESS

Citation: Saitta P. (2021) Un cuscinetto chiamato informalità. Politiche, simboli e materialità di un "segreto", in "Cambio. Rivista sulle trasformazioni sociali», Vol. 11, n. 21: 51-64. doi: 10.36253/ cambio-10825

Copyright: (c) 2021 Saitta P. This is an open access, peer-reviewed article published by Firenze University Press (http://www.fupress.com/cambio) and distributed under the terms of the Creative Commons Attribution License, which permits unrestricted use, distribution, and reproduction in any medium, provided the original author and source are credited.

Data Availability Statement: All relevant data are within the paper and its Supporting Information files.

Competing Interests: The Author(s) declare(s) no conflict of interest.
Monographic Section

\section{Un cuscinetto chiamato informalità. Politiche, simboli e materialità di un "segreto"}

\author{
Pietro Saitta \\ Università di Messina \\ pisait@gmail.com
}

\begin{abstract}
This essay explores the semantics of the term "informal economy" and, in particular, the relation between the two words. It shows how this subject is anything but something with no form and no structure. Moreover, it reflects on the historical relations between this type of economy and the cyclical transformations of capital. Informal economy is seen as a buffer that makes such changes both socially bearable and symbolically necessary - in order to produce alliances between classes and mobilize different sentiments in given circumstances. The example of (neo-)populism(s), especially in a Southern Italian city (Messina), is provided and shortly discussed. Finally, it advocates descriptions of the phenomenon that unfold the substantial overlapping of what is official and "secret", and is in fact part of the experience of millions of people in an endlessly changing world.
\end{abstract}

Keywords: informal economy, neo-populism, ideology, mezzogiorno (south italy), precarity.

\section{INTRODUZIONE}

Con un po' di irriverenza, si potrebbe forse dire che ciò che oggi rimane di interessante del concetto di «economia informale» è l'aggettivo. Di cosa sia questa economia, infatti, si è scritto moltissimo a cavallo tra gli anni settanta del Novecento - quando l'espressione, già presente in un volume di Doeringer e Piore (1971: 246), divenne celebre per merito di un seminale articolo di Hart (1973) - e il presente (per delle rassegne limitate alle sole pubblicazioni in inglese, che escludono dunque la miriade di testi e prospettive offerte in lingue differenti, si vedano: Losby et alii 2002; Hansen e Vaa 2004; Mukhija e Loukaitou-Sideris 2014). Di questo oggetto - l'economia informale - si sono occupate istituzioni nazionali e internazionali per la ricerca e la regolazione in materia di economia e lavoro (primo tra tutti, l'International Labor Office), tecnici e consulenti (talvolta destinati a una straordinaria fortuna internazionale, come nel caso di Hernando De Soto); e poi 
economisti mainstream, antropologi (fu dopo tutto uno di loro a rendere famosa l'espressione), geografi, sociologi urbani e militanti politici, per lo più di sinistra. Senza dimenticare i criminologi "critici" o quelli "amministrativi".

Per lo meno in ambito accademico, si tratta dunque di un tema e di un'espressione fortunatissima, che ha finito con l'essere adottata da una pluralità di discipline per quel che racchiude e per ciò a cui può essere associata. L'economia informale è infatti l'insieme di quei modi di produzione, lavoro e reddito che sfuggono alla regolazione statale e alla visibilità delle agenzie di controllo (Hansen e Vaa 2004). Costituisce uno spazio ibrido e interclassista in cui convivono lo sfruttamento del lavoro e l'evasione fiscale a opera di imprenditori di peso e condizioni diverse, che possono agire nella più completa irregolarità così come in un terreno misto, che combina illeceità e conformità (Shapland 2009). Teso, cioè, alla produzione di beni o all'erogazione di servizi che possono essere in sé leciti, ma irregolari per le modalità di produzione; oppure del tutto illegali (Losby et alii 2002). In termini più concreti, l'espressione economia informale indica tanto gli spazi ricavati da ricchi imprenditori al fine di eseguire manovre che sfuggano alla tassazione quanto quelli in cui si compie la sussistenza di soggetti poveri, sprovvisti di significativi capitali economici o culturali, che da attività di scarso valore ricavano quanto basta per sopravvivere malamente all'interno di un mercato del lavoro segmentato (Ferman et alii 1987; Berger e Buvinic 1989; Rajiman 2001).

Se questo è grosso modo il quadro racchiuso dall'espressione, è prevedibile che l'economia informale sia divenuta nel tempo anche il centro di discorsi, preoccupazioni e politiche incentrate sui costi sociali dell'evasione fiscale, sulla concorrenza nel commercio, sulla sicurezza del lavoro, sui diritti delle persone e sulla protezione ambientale. Ma anche - e forse soprattutto - sulla pubblica sicurezza (come nel caso dello spaccio di sostanze stupefacenti e il lavoro sessuale) oltre che l'ordine e l'estetica urbana (è il caso di quei centri storici assediati, come vuole un certo linguaggio pubblico stereotipato diffusosi negli anni, da bancarelle abusive che avrebbero conseguenze sulla viabilità o l'ordine percepito delle strade). Sintetizzando quanto sin qui detto, l'economia informale può essere descritta anche come come la quota della ricchezza sottratta allo Stato; una misura dei costi sociali relativi alla salute dei lavoratori e all'ambiente; un indicatore dei livelli di criminalità presenti in una società e una variabile interveniente nel farsi della sicurezza; un proxy dei livelli percepiti di disordine e dell'efficacia della regolazione e del controllo negli ambienti urbani; e, infine, un modo di sussistenza.

\section{SEMANTICA DELL'INFORMALE}

In apertura a questo saggio si è osservato che è l'aggettivo - informale - a mostrare ancora degli aspetti interessanti e relativamente meno esplorati rispetto al complesso concettuale dato dall'espressione «economia informale». La letteratura (e in primo luogo Hart, che ne decretò il successo) non fornisce spiegazione per la scelta di questo aggettivo, che infatti non risulta generalmente mai troppo chiaro per chi è esterno al dibattito scientifico e, almeno di primo acchito, fatica a coglierne il significato quando lo sente accostato all'economia. Nel noto studio di Hart (1973: 61) l'espressione compare nelle primissime righe e indica l'effetto che inflazione, bassi salari e la crescente richiesta di specializzazione o titoli di studio hanno avuto sulle attività del sottoproletariato ghanese, e non solo quest'ultimo, spingendolo verso forme sovrapposte e autonome di lavoro utili a sostenere il reddito. Possiamo dunque immaginare che Hart ricorra a questo aggettivo, «informale», per denotare il carattere non contrattualizzato - per l'appunto, non formalizzato - dei rapporti lavorativi, oppure la mancata registrazione e la relativa «incensibilità» delle attività imprenditoriali escogitate dalla popolazione. Insomma questa scelta lessicale dovrebbe ricalcare o essere ispirata dagli usi giuridici del termine «formale», per i quali indica, tra le altre cose, il rispetto delle procedure e della loro forma. Negli impieghi successivi fatti da altri autori la medesima espressione viene talvolta usata per distinguere le attività e le relazioni lavorative non regolari da quelle propriamente criminali. A tale riguardo può essere utile osservare come, nell'analisi del fenomeno in oggetto, non risulti secondario il ruolo della lingua impiegata per le analisi e per le comunicazioni scientifiche, lì ove le espressioni prescelte per discutere del tema presentano talvolta sfumature di significato non immediatamente apprezzabili per individui allofoni, oppure non propriamente interni ai particolari codici linguistici. È questo il caso di apparenti sinonimi come, per esempio, l'espressione underground economy, che in inglese può effettivamente includere il significato di informale come 
irregolare, ma veicola più facilmente il senso di un'economia di tipo criminale (Losby et alii 2002: 8). In italiano, viceversa, economia «sotterranea», «irregolare», «nascosta», «in nero» possono essere adoperati come perfetti sinonimi di informale e appaiono forse persino più comprensibili.

Ragionando sugli impieghi di «informale» si possono prospettare dunque due ipotesi. La prima è quella non vi sia stato un eccessivo ragionamento sull'accostamento tra sostantivo e aggettivo, dacché la combinazione, all'interno del sistema linguistico in cui nasceva, sembrava convogliare sufficientemente il senso di un insieme di rapporti produttivi oppure lavorativi non ufficiali e invisibili dunque alle amministrazioni. La seconda è che nella ricezione in altre lingue, e in certi ambiti disciplinari più che in altri (possiamo presupporre che ciò avvenisse con relativa facilità in economia), l'aggettivo informale risultasse suggestivo anche perché richiamava l'idea di ciò che non ha forma o autentica struttura. È dunque possibile immaginare che nel concetto di economia informale sia implicito un regime "scopico", relativo cioè alle modalità dell'osservazione scientifica, e anche un giudizio di valore, che, almeno all'origine delle teorizzazioni in materia, concepiva questa tipologia di occupazioni come un indistinto. Ossia un oggetto problematico, che assolveva delle funzioni sociali ed economiche, ma la cui conoscenza intima, nei termini del suo funzionamento, restava un compito tutto sommato inessenziale. Tutt'al più limitato agli scopi di certe discipline, ma non di altre, che potevano invece accontentarsi della "sostanza" confinata, per esempio, nei verbali di polizia relativi ai sequestri di merce o ai fermi di persone coinvolte in attività irregolari, oppure alle stime condotte dalle agenzie di controllo economico. E che per questo hanno potuto a lungo fare a meno di considerare come - quando è visto in azione, all'interno delle località e da vicino - l'informale abbia quasi sempre una struttura, delle funzioni sociali, degli attori, una logica e delle gerarchie. E sia, dunque, tutt'altro che indistinto, privo di forma o acefalo per natura. Una consapevolezza che si è imposta probabilmente nei saperi di polizia prima che in quella degli economisti strictu sensu e che nel tempo ha rafforzato l'idea che l'informale sia una questione essenzialmente criminale, para-criminale $\mathrm{o}$, comunque, di malaffare. Conducendo così alla trasformazione di una questione sociale in un fatto essenzialmente penale.

Un esito tutt'altro che scontato se si osserva per esempio l'esperienza latino-americana ove - entro cornici che coincidevano a volte con le forme locali del nascente neoliberismo e, in altre, con l'autorganizzazione sottoproletaria - l'informale è divenuto una risorsa e un modo di accesso a forme di emersione di un nuovo soggetto «cittadinario», che proprio attraverso l'informalità del lavorare e del vivere ha maturato un nuovo orgoglio e un nuovo senso politico da assegnare alla propria esistenza e alle proprie pratiche (De Soto 2000; Holston 2009; Coletto 2010). Ciò che non a caso, nel parlare di queste stesse esperienze e paesi, ha indotto altri osservatori - talvolta eccessivamente "romantici" secondo alcune critiche (Abu-Lughod 1990; Ortner 1995) - ad accostare la nozione di informale a quello di «resistenza» (Scott 1990), così come si definisce l'insieme dei processi popolari disarticolati e spontanei di opposizione ai «progetti» (volti per esempio alla costituzione di gerarchie sociali, di modelli urbanistici e di forme di spazializzazione delle attività umane) e ai «regimi visuali» (di tipo fiscale, anagrafico e "di polizia”) perseguiti dagli stati moderni nel corso della loro formazione (Scott 1998), con intenti e modalità giudicate dalle popolazioni o dagli osservatori stessi oppressive, coloniali o classiste.

Se queste ipotesi sono plausibili, e se considerazioni almeno in parte compatibili con esse hanno guidato i processi di regolazione ed emersione delle economie irregolari in molti paesi latino-americani (si vedano ancora una volta i casi ispirati da De Soto), si può altresì sostenere che nella vicenda europea e nordamericana i giudizi essenzialmente destituivi del fenomeno, propri degli esperti maggiormente influenti e attivi nella consulenza politica rinvenibile nel linguaggio delle discipline economiche così come nelle sue traduzioni, adattamenti e ricezioni in contesti disciplinari e nazionali diversi - sia facilmente transitato in altre istituzioni e livelli facendosi dominante: per esempio nel mondo della politica, in quello tecnico-istituzionale responsabile delle politiche urbanistiche, nella cultura professionale dei burocrati di strada e, da qui, con gradualità, nel senso comune degli operatori dell'informazione e della società in genere.

L'idea insomma è che i termini «informale», «clandestina», «sommersa», «nascosta» etc., affiancati al concetto di economia, abbiano funzionato come detonatori di stigmi che hanno finito col travolgere le attività e, soprattutto, le persone coinvolte. Generando, com'è probabilmente banale a dirsi, associazioni semantico-simboliche legate al segreto e a un mondo invisibile che affianca quello ufficiale e che trama ai suoi danni (Brighenti 2010: 65). 


\section{LE RISTRUTTURAZIONI DELL'ECONOMICO}

Certo, non bisognerebbe essere ingenui e occorre leggere questi passaggi nel flusso storico. Quel flusso che a partire dagli anni Settanta del Novecento vede il dibattito pubblico italiano, così come quello di molti paesi dell'Europa occidentale, essere attraversati dalla progressiva centralità della "piaga" dei doppi lavori, dell'evasione fiscale e dell'impegno di molti cittadini a tenere nascoste parti della propria ricchezza e delle sue fonti (Gallino 1975; Gutmann 1978; Alden 1981). È utile, a tale proposito, ricordare come sia stato proprio il settore «informale» a coadiuvare l'accelerazione degli anni Sessanta e il cosiddetto boom economico (Crainz 2005; Toffanin 2016). Ciò che ha fatto di questo mondo parallelo, informale, «segreto» e invisibile un elemento strutturale dell'economia italiana (e non solo questa. Un'affermazione, del resto, che risulterebbe vera sia con riferimento al periodo del dopoguerra, sia alla storia del capitalismo in ogni stagione. Si vedano a riguardo: Wallerstein 1983; Broad 2000). Un mondo «segreto», dunque, che a partire dalla ricostruzione post-bellica ha assunto un carattere di massa e ha connesso tra loro milioni di persone accomunate dalla volontà, o forse dalla necessità, di rendersi invisibili allo sguardo delle agenzie pubbliche. All'interno di questa cornice strutturale, la storia degli ultimi due o tre decenni (quelli cioè delle politiche di rientro dal debito, della flessibilizzazione del mercato del lavoro e dell'austerità, che hanno orientato il processo europeo e imposto nuovi vincoli ai paesi aderenti; in particolare a quelli maggiormente "indisciplinati" come l'Italia) può essere letta come quella della lotta per l'emersione. Una battaglia incentrata da un lato sull'intensificazione delle attività ispettive e della repressione e, dall'altro, sulle attività «promozionali», volte ad agevolare la regolarizzazione delle attività non dichiarate (Esposito 2012: 6 passim).

E sempre a proposito di questo flusso storico, tra gli anni Settanta del secolo scorso e il presente sono intervenuti alcuni rilevanti cambiamenti. Il doppio lavoro, un fenomeno che in quegli anni ormai lontani si diceva riguardasse anche soggetti impiegati nella pubblica amministrazione, non è più un tema del giorno; per quanto questo sia tutt'altro che scomparso e, anzi, riguardi oggi una vastissima popolazione di nuovi precari, spesso sottopagati e letteralmente costretti a collezionare più lavori. Ciò in ragione dei bassi salari, ma anche della ristrutturazione del mercato immobiliare e del costo della casa. Un carico spesso insostenibile di spese che, oltre alle classiche valenze economiche, aggiunge al concetto di informale nuovi significati connessi all'abitare, che si fa esso stesso irregolare. Ossia caratterizzato dal ripetuto susseguirsi di sfratti, da occupazioni di immobili e da forme comunitarie di condivisione e convivenza molto diverse da quelle borghesi - in case e appartamenti "monofamiliari" o individuali - venutesi a strutturare nei suoi termini di massa a partire dal dopoguerra (Fregolent e Torri 2018; Esposito e Chiodelli 2020; Pozzi 2020). Un esercito dunque di doppi o tripli lavoristi, spesso residenti in spazi non convenzionali, spinti dal fine non di accumulare risparmio da sottrarre al fisco, ma di sopravvivere. Lavoratori che frequentemente sommano impieghi cattivi ma regolari - utili ad accedere agli imprescindibili sussidi necessari a navigare i periodici momenti di inattività - e lavori in nero, indispensabili per integrare entrate solitamente molto magre. Persone, peraltro, che si muovono entro aspirazioni e proiezioni individuali sempre più limitate dal punto di vista temporale, fino a coincidere col semplice quotidiano o quasi (Murgia 2010; Giannini, 2017: 109; Carlini 2019). Qualcosa che appare compatibile, del resto, con un'economia ufficiale ormai convertita ai servizi e al digitale, che aspira a rendere i "lavoretti" - in primis quello di rider o, meglio, fattorino, pagato pochi spiccioli all'ora, soggetto al capriccio degli algoritmi e dei manager, bici- o auto-munito, senza assicurazioni né garanzie, in lotta col tempo, che diventa la sua risorsa più preziosa e la sua croce, dacché è dalla velocità delle consegne che dipende la possibilità accordatagli di lavorare - una prospettiva accettabile d'impiego (Bowe et alii 2000; Crouch 2019; Tassinari e Maccarrone 2018). Una economia ufficiale, dunque, che diventa spesso indistinguibile da quella informale; ossia che retribuisce e non offre garanzie allo stesso modo della seconda. Al centro di questi processi, inoltre, non vi sono più soltanto soggetti pienamente autoctoni, ma anche gli immigrati. Questi ultimi, peraltro, precarizzati secondo logiche segmentarie, che distinguono tra stranieri regolari di lunga permanenza (relativamente "garantiti" dall'espulsione o dalle manifestazioni estreme della marginalizzazione, ma mai abbastanza), tra immigrati illegali (invisibili ed esposti a ogni tipo di insidia proveniente da datori di lavoro o polizie) e, infine, tra richiedenti asilo, rifugiati e "protetti" il cui status è sì legale, ma limitato nel tempo e perciò a scadenza (quel che determinerà la «clandestinizzazione» di molti di essi) (De Genova 2002; Mezzadra e Neilson 2013; Bolzoni et alii 2015; Sanò 2018). 


\section{IDEOLOGIE}

In questa cornice ad apparire «informale» - ossia irregolare, parzialmente irregolare e apparentemente «indistinta», per recuperare un'espressione usata in precedenza - è una parte consistente della società italiana, con intensità mutevoli il cui grado dipende dall'intersecarsi e configurarsi di caratteristiche quali la classe, l'età, la nazionalità e le fortune individuali. Per quanto nella comprensione di queste variabili occorra forse essere meno netti di quanto le classiche letture del passato potrebbero indurre a fare. Se il mondo subalterno tradizionale quello delle occupazioni malpagate, sporche e faticose - si accompagnava a una origine e a una condizione fatte di bassa scolarità e riproduzione della povertà, il nuovo mondo precario appare spesso fatto di mobilità verticali negative. Ossia di riproduzioni sociali mancate, che vedono molti soggetti appartenenti alle classi medie emerse dal dopo-guerra uscire frequentemente dalla propria condizione originaria per avvitarsi nel vortice dell'instabilità, forti di un capitale simbolico e culturale a cui non corrisponde però un capitale economico adeguato. Una storia questa fatta di individui, ma anche di interi settori, che vedono divorziare l'allure simbolico - ossia la fama e il prestigio connessi per esempio all'industria culturale e ai suoi professionisti - e il dato materiale dei redditi reali e dei fatturati (Ventura 2017).

Ciò che si trae da questa descrizione è che malgrado la vocazione al patinato di molta della cultura popolare, la società italiana nel proprio complesso sia ampiamente, e forse maggioritariamente, divenuta «informale», ossia precaria e impoverita; ma non è per questo acefala, priva di direzione e incomprensibile. Infatti i nuovi processi produttivi, ideologici e giuridici relativi al lavoro, così come chi li ha messi in moto e li dirige, risultano nel complesso abbastanza chiari. Traggono origine dalla fine della centralità del lavoro come valore e come soggetto politico collettivo; dal progressivo svanire delle ideologie del conflitto e della libido associata alle lotte; dall'erosione di ordine culturale del valore sociale assegnato al sindacato; dalla capacità egemonica del lusso; dai meccanismi oggettivi e di tipo culturale innescati dal debito al consumo; dal progredire di ideologie essenzialmente neo-corporative che assegnano importanza all'impresa e agli imprenditori molto più che al lavoro dipendente; dal tramontare di quelle capacità di lettura della politica che classicamente legava le appartenenze agli interessi materiali e di classe.

È questo un elenco parziale e incompleto di elementi ideologici od oggettivi, strutturatisi oppure destrutturatisi nell'arco di un quarantennio o poco più, che si è tradotto in un intreccio di disuguaglianza strutturale e contraddizioni politico-culturali, corrispondenti più o meno a ciò che un tempo si sarebbe chiamata «falsa coscienza». Un processo, insomma, che vede la «sovrastruttura» giocare un ruolo fondamentale per il mantenimento dell'equilibrio e dell'ordine. Tuttavia accanto a questo, e prima di qualunque analisi del sovrastrutturale, bisognerebbe anche ricordare che se gran parte delle riforme del lavoro, così come dei tagli al welfare, sono passate nel silenzio generale oppure in un'opposizione che ha riguardato parti tutto sommato minoritarie del mondo del lavoro o della società italiana, questo lo si deve in parte al fatto che una quota consistente di essa era esterna al lavoro regolare, alla rappresentanza sindacale e a molte forme di protezione sin dall'avvio della storia repubblicana (Ginsborg 1989; Ferrera 2006; Crainz 2012). Le disuguaglianze che a partire dalla fine degli anni Novanta dello scorso secolo avevano iniziato a colpire i garantiti, così come i nuovi processi di precarizzazione, non erano né estranee né distanti dall'esperienza quotidiana di una parte consistente della società italiana rimasta esclusa dall'occupazione nel settore pubblico. Pertanto tale evenienza non metteva in moto nessun particolare sentimento reattivo o solidale in quella parte del paese che precaria e poco garantita lo era già e che avrebbe continuato a restare tale anche dopo le riforme del mercato del lavoro. Inoltre estese aree geografiche e sociali del paese, concentrate per buona parte nel Mezzogiorno, erano soggette a forme di sudditanza clientelare, volte a barattare voti e protezione sociale, che avevano come referenti personalità e partiti egemoni nei quadri politici succedutisi nel corso del tempo. Erano perciò settori sociali intrappolati in una economia della promessa, generalmente fondata sulla procrastinazione del beneficio atteso e, dunque, sull'attesa paziente e docile (Allum 1975; Chubb 1983; Fantozzi e Raniolo 2018; Farinella e Saitta 2019).

$\mathrm{Ma}$ al netto di quanto appena osservato, e recuperando un lessico ormai decisamente passato, si potrebbe osservare che nell'informalizzazione della società italiana un ruolo non secondario devono averlo giocato gli «apparati ideologici di stato» (Althusser 1976); o, magari, quel complesso stato-impresa che è diventato centrale nell'Italia di Berlusconi, ma che è in realtà frutto del matrimonio tra comunicazione pubblica e interessi privati che ha interessa- 
to l'Europa e gli Stati Uniti a partire dagli anni ottanta del secolo scorso in modo abbastanza uniforme (esemplare il caso del gruppo Murdoch, che, a testimonianza della natura transcontinentale della tendenza di cui discutiamo, ha origini in Australia e da lì si diffonde nel resto del mondo). Questi apparati ideologici - in particolare quelli televisivi o digitali, fondati sull'intrattenimento, la trasformazione dell'informazione in spettacolo e sull'emergere di figure ibride di intellettuali «intrattenitori organici» - hanno giocato un ruolo imprescindibile nel creare le forme di soggettività collettive descritte in precedenza: la soggettività, si potrebbe dire, del disimpegno e del narcisismo di massa. Si tratta, insomma, del tipo di presenza nello spazio pubblico che è stata premessa fondamentale per il consolidarsi di quella impotenza politica collettiva che ha accompagnato le asimmetrie e le disuguaglianze strutturali sin qui descritte. Una condizione che, in differenti fasi, si è tramutata in disinteresse verso il politico, oppure in indignazione verso questo stesso universo; o in un sentimento che sublimava la rabbia, trasformandola in desiderio per la merce. O, ancora, che progrediva illudendo il soggetto medio "integrato" che i vecchi ordini e gerarchie stessero sopravvivendo; e che egli, nonostante tutto, fosse collocato su un livello diverso dall'ultimo. Per quanto si percepisse anche in una posizione precaria ed esposta al rischio dell'arretramento, oltre che personalmente vulnerabile alle minacce alla sicurezza fisica e lavorativa provenienti da una società che cambiava intanto pelle e colore facendosi gradualmente meno familiare. In questo quadro la pubblicità della merce si alternava allo spettacolo della morte violenta per mano dello straniero. E formava una dimensione di stupore, o awe, a cui si accompagnavano anche le trasformazioni del linguaggio e la sua radicalizzazione, estendendo così le frontiere del dicibile. Per l'appunto, il riemergere nella cultura italiana del vecchio linguaggio dell'«amico e del nemico», che negava l'altro «radicale» (per esempio l'immigrato e il fondamentalista islamico), ma anche quello «prossimo» (il tossicodipendente, l'incivile e la zecca comunista dei centri sociali; ma anche il professore universitario, il radical-chic e il membro della "casta") (Panarari 2010; Binik 2017).

Bourdieu (1993), com'è noto, ha chiamato tutto questo la «miseria del mondo»: più precisamente, la miseria di posizione di chi vede un vecchio mondo e le sue sicurezze venire giù; oppure che vede nuove minacce profilarsi all'orizzonte, ma non ha nomi per chiamarle. E che perciò può solo ricorrere al linguaggio di quelle «idee senza parole» (Jesi 1979) che si richiamano alla Tradizione, alla Terra, alla Sovranità etc. Ma questa è in fondo anche la lingua della «violenza strutturale» (Farmer 2004): un tipo di violenza che esiste anche quando nessuna la esercita sul corpo e che investe il povero assoluto così come il piccolo-borghese sulla via del tramonto. Entrambi attori in preda a dolori e passioni, le quali, pur implicando asimmetrie di posizione, denotano anche la riduzione della distanza che separa i soggetti (per inciso una dinamica analoga al rapporto tra colonizzati e colonizzatori nelle terre occupate, almeno secondo Memmi 1979; una relazione che vale la pena ricordare qui, sia pure fugacemente, perché anticipatrice delle dinamiche successive del capitalismo europeo dopo il suo "rimpatrio"). Se la violenza strutturale è dunque quella che viene esercitata in modo indiretto, che non ha bisogno di attori per essere eseguita in quanto è prodotta dall'organizzazione sociale stessa, e che si traduce in patologie, è facile vedere come dietro il malessere della vita nel nuovo capitalismo di cui ha parlato recentemente Fisher (2009) debba starci quella crescente informalizzazione del sociale che è al centro di questa stessa rassegna.

Un male, a ogni modo, che deve essere iscritto nella struttura dell'oggetto e che infatti accomuna tanto il nuovo quanto il vecchio capitalismo. Incidentalmente ci si può chiedere infatti se differiscano così tanto tra loro la depressione e la passione per gli psicofarmaci nella società neoliberale, discusse da Fisher, e la nozione di «anomia» proposta da Durkheim (1893) al fine di interpretare quelle patologie sociali legate alla nuove forme di normatività e appartenenza, così come al lavoro e all'economia, che andavano dispiegandosi sotto i suoi occhi negli anni a metà tra Otto e Novecento.

\section{MEMORIE E AMNESIE}

Non costituirebbe d'altronde una novità osservare che il capitalismo presenta la caratteristica di mutare - talvolta in modo consistente - mantenendo però anche forme sorprendenti di continuità e, in particolare, di continuità nelle risposte alle trasformazioni, spesso socialmente dolorose, dettate dai processi di ristrutturazione. Sarebbe 
del resto la stessa economia informale una riprova di questo, dal momento che la «casualizzazione» del lavoro ovvero la ciclica assunzione di caratteristiche irregolari rispetto a quelle dominanti in ciascuna fase - è una costante del capitalismo storico nei momenti di crisi (Sassen 1982; Broad 2000: 24). Coerentemente con questa osservazione - ma su un piano questa volta sovrastrutturale - la storia del capitalismo appare anche come un fatto di ripetizioni (reali o immaginarie) e, soprattutto, di repertori e risposte culturali sedimentate, brandite ritualmente dinanzi all'avanzare di minacce che spesso sono anch'esse inedite e vecchie allo stesso tempo. $\mathrm{O}$, magari, interamente nuove; ma a cui, consolatoriamente, si risponde con strumenti familiari, depositati nella memoria e nelle sue «amnesie strutturali».

Accantonando per un po' il nostro oggetto - l'informale - col fine di indagare il contesto morale entro cui questo si sviluppa, comprendiamo forse meglio la ragione per cui la memoria e le amnesie appaiono importanti se riflettiamo sul fatto che gli anni intorno a cui scriveva Durkheim erano esattamente quelli in cui si affacciava uno scandalo e, soprattutto, una "questione" destinata a caratterizzare tutto il secolo seguente e anche quello corrente sino a questo momento: sono cioè gli anni dell'Affaire Dreyfus, dell'emergere della nuova questione ebraica e di nuove forme di divisione sociale che daranno vita a «racconti sull'altro» e all'individuazione di capri espiatori a cui imputare le colpe di gran parte di ciò che va male nel mondo (Harris 2007). Un processo, per l'esattezza, che ha la sua vera origine in un periodo precedente (in quei secoli 1500-1700 in cui rinasce e si placa momentaneamente la «caccia alle streghe»; quello che è in realtà un sinonimo della persecuzione nei confronti di molte altre forme di alterità del tempo, che includevano eretici, ebrei, valdesi e altri irriducibili all'ordine) (Trevor-Roper 1969), ma che per economia dello spazio tralasceremo, limitandoci a rievocare velocemente l'ultimo secolo di storia o poco più. Sono gli anni, quelli a cavallo tra Otto e Novecento, in cui l'ebraismo riprende a incarnare il male, l'occulto e la congiura contro il mondo. È di quegli stessi anni, infatti, anche un testo destinato a fare la storia dei racconti sul complotto ebraico: I protocolli dei Savi di Sion. Com'è noto, l'Affare Dreyfus e i Protocolli sono il preludio della circolazione di idee sui portatori del male e del disordine che saranno parte integrante di un clima politico e morale e che, insieme ai processi di ristrutturazione economici e geopolitici determinati dalla Prima Guerra Mondiale, condurranno verso le esperienze naziste e fasciste e la sperimentazione di forme di coesione basata sull'alleanza tra classi sociali nel contenimento di altre classi. Sono queste esperienze che, nel corso della loro evoluzione, produrranno a propria volta nuovi sentimenti, linguaggi, contaminazioni e sperimentazioni tecniche orientate alla conquista dell'egemonia dall'ovvio carattere autoritario, razzista e «populista», destinati a durare e sedimentarsi ben oltre la vita dei suddetti regimi. Una "ricca" stagione ideale, però, che è preceduta da una grande confusione e dalla circolazione di ulteriori suggestioni a opera di altri autori - e che da lì a poco verranno assimilate anche dai nascenti regimi - tese a lasciarsi alle spalle l'alternativa tra destra e sinistra. Negli anni dell'occupazione della Ruhr e delle riparazioni di guerra, l'appello di Karl Radek per un fronte unito dei lavoratori, aperto ai ceti medi e alla piccola borghesia patriottica e capace di difendere l'indipendenza del Paese dall'imperialismo straniero, segnava l'inizio di una guerra d'egemonia destinata a segnare l'avvento del populismo contemporaneo e persino del "rosso-brunismo" (come si definiscono oggi le variegate aspirazioni di una composita galassia di movimenti a un ordine nuovo, posto oltre le categorie politiche classiche, secondo un bricolage che coniuga socialismo, capitalismo e identitarismi regionalisti o nazionalisti) (Azzarà 2018).

La tesi qui esposta è che tutto questo costituisca un archivio delle memorie e delle amnesie proprie di una cultura italiana ed europea, che, nelle stagioni di crisi profonda, si riattivano alla stregua di un automatismo, inquietando e rassicurando insieme l'«individuo-massa» smarrito e alla ricerca di un senso; ossia di ciò che è noto, "logico" e alla portata della sua comprensione perché seppellito nelle strutture intime della sua cultura.

Limitando lo sguardo all'ultimo secolo e mezzo o poco più, e continuando a tralasciare per motivi di spazio il farsi dei fenomeni di regalità e di «costruzione dei popoli» su archi di tempo più lunghi e autenticamente costitutivi dei sentimenti politici nella modernità (come fanno, per esempio, i saggi contenuti in Jensen 2016; ma il problema, su scale temporali differenti, è in fondo lo stesso di Weber, relativo a cosa resti della democrazia degli antichi nel mondo modernizzato. Si veda su questo: De Federicis 2017), è abbastanza frequente che nelle stagioni di ristrutturazione capitalista e stravolgimento degli equilibri di classe consolidati, la tendenza al leaderismo, gli identitarismi localistici (ossia etnici, nazionalisti, regionalisti), la nostalgia revanchista per epoche precedenti di 
grandeur o supposto benessere, l'avversione verso capri espiatori interni ed esterni alla nazione e le "teorie del complotto" emergano molto rapidamente nella forma di sentimento popolare e di politiche (senza, tuttavia, che vi sia un necessario ordine di precedenza tra i due. Per quanto i sentimenti sono più facilmente prodotti e organizzati dal mondo politico). Se questi sono in realtà processi e tic molto antichi, possiamo ciò nonostante dire che nell'età contemporanea, in Italia ed Europa, sono gli autoritarismi di destra ad avere scritto la grammatica sentimentale politica popolare e spontanea. Tanto nel senso che esistono una miriade di formazioni che si richiamano esplicitamente alle esperienze naziste e fasciste, quanto in quello per cui le ossessioni e le tendenze di coloro che non si richiamano immediatamente a quelle ideologie sono comunque oggettivamente compatibili con esse. Oltre che facilmente rievocabili se si interloquisce con chi detiene tali compatibili convinzioni.

\section{INFORMALITÀ E “(NEO-)POPULISMI”}

A organizzare questi automatismi nell'Italia contemporanea - ma anche in differenti paesi europei e negli Stati Uniti, con sfumature e adattamenti che dipendono dalle storie nazionali - è un insieme di forze che possiamo definire «populiste»o, meglio, «neo-populiste». Sono entrambi termini insoddisfacenti, irrispettosi delle differenze tra posizioni rinvenibili in campo e "acchiappa-tutto", che in questa discussione impiegheremo nell'accezione aperta proposta da Canovan (1981). Il «populismo autoritario» indagato dall'autrice investe infatti una dimensione sentimentale, che ruota attorno alla percezione di sradicamento esperito dall'individuo e dalle masse nelle fasi di mutamento radicale. Un sentimento a cui gli imprenditori politici rispondono con una proposta di riscatto che viene da loro stessi; o, meglio, dal leader. Con Laclau (2008) e Stanley (2008) possiamo dunque parlare dei fenomeni neo-populisti come dispositivi retorici volti a costituire identità collettive fondate sull'antagonismo del binomio «popolo-leader» - quel complesso che incarnerebbe la virtù, il bene, la moralità e il lavoro - nei confronti di élite predatrici e altri nemici interni ed esterni che assediano il primo polo di questa contrapposizione.

Se la dimensione nazionale del populismo è quella che di solito attira su di sé l'attenzione dei commentatori (Blokker e Anselmi 2020), è pur vero che da tempo va emergendo una dimensione urbana e iper-localistica del fenomeno, che adatta i grandi temi nazionali o globali, insieme alle tecniche e alle tecnologie utili all'affermazione della propria egemonia. Per esempio attraverso l'uso dei dialetti anziché della lingua nazionale, oppure la mobilitazione di simboli che includono il territorio, il cibo o qualsiasi altro elemento in grado di evocare l'«identità» locale e le relative contrapposizioni. I populismi, inoltre, sono anche un fatto di alleanze tra classi (Germani 1978). Nella storia del movimento fascista, per esempio, appare centrale la relazione tra nobiltà, alta borghesia e classi medie ai fini della promozione di una mobilitazione dall'alto finalizzata al controllo delle classi subalterne. Mentre nel caso del nazional-populismo peronista prevale un blocco sociale formato dalla classe media latino-americana integrata con le classi subalterne (Anselmi 2017: 9). Se la storia dei populismi italiani contemporanei di scala nazionale (quelli incarnati da Lega, Movimento 5 Stelle, Berlusconi e Renzi) è prevalentemente una vicenda di mobilitazione delle classi medie (Biancalana 2020), che in alcuni casi vede però un attivo coinvolgimento di settori dell'alta borghesia, i livelli urbani appaiono più articolati.

Lo spunto per delle riflessioni in merito al tema delle alleanze populiste, insieme a un'analisi delle connessioni di quanto sin qui osservato con l'economia informale o con l'informalizzazione del sociale, è dato un caso siciliano: quello di Messina e del suo Sindaco, Cateno De Luca. Quest'ultimo è un politico di professione dalla carriera trentennale, cresciuto inizialmente nel cono d'ombra della Democrazia Cristiana e confluito successivamente in una miriade di formazioni, legate all'autonomismo siciliano così come a Forza Italia. Nel corso degli anni è stato sindaco di villaggi, di paesi di poco più grandi e, finalmente, di una città metropolitana. Ma è stato anche consigliere comunale e membro del Parlamento della Regione Sicilia. È inoltre un "imprenditore" che ha fatto la sua fortuna con i centri di assistenza fiscale - una forma di capitalismo assistito che si sviluppa nella cornice dei servizi allo Stato - e finalmente ha preso a operare nel settore delle banche dati. La sua federazione di Caf (Fenapi) e la sua impresa digitale hanno sedi in tutta Italia e una rappresentanza a Bruxelles. De Luca è stato il parlamentare regionale più ricco; inoltre dalla sua ultima dichiarazione dei redditi si evince che è milionario e che la sua ricchezza è 
cresciuta negli anni della sindacatura in modo ragguardevole. Ciò nonostante è figlio di contadini di un paesino della provincia messinese. Rivendica le sue origini povere e rurali e si esibisce costantemente in dirette Facebook che hanno per sfondo la casa natia. Un'abitazione che trasuda umiltà e decadenza. Dagli arredi economici alle vistosissime macchie di umidità sui muri, passando per l'impiego di cappelli di lana con vistose bruciature di sigaretta, esibiti nel corso di passeggiate che hanno per sfondo ambientazioni rurali (molto simbolicamente, il percorso che ogni mattina lo conduce dalla sua abitazione a quella dei genitori per un saluto prima dell'inizio delle attività amministrative), la rappresentazione che di sé fornisce il milionario Cateno De Luca è fondamentalmente ispirata al pauperismo, all'autenticità, alla famiglia e al culto delle origini (per una più ampia fenomenologia del personaggio: Saitta 2020).

In una città che conta ormai meno di 230.000 abitanti, che da tempo vede in media emigrare annualmente circa 1.500 residenti, in cui il $42 \%$ della popolazione è priva di reddito e in cui il $33 \%$ dei contribuenti dichiara redditi compresi tra 0 e 10.000 euro annui lordi (ossia tra 0 e 800 euro mensili lordi); e in cui il $40 \%$ è compreso tra 15.000 e 26.000 (e quindi tra 1.200 e 2.200 euro mensili lordi) (Limosani 2021), l'alleanza promossa dal ricco Cateno De Luca ha per controparte la borghesia minuta del commercio, dell'artigianato e l'altra, spesso di poco superiore, della rendita immobiliare (questo tipo di proprietà costituisce il 50\% degli investimenti e della ricchezza cittadina). Se sul piano culturale il suo linguaggio intriso di dialettismi e dal registro basso se non infimo (fatto di urla, insulti rivolti contro i «blasonati» e rancore) è idoneo a connetterlo sentimentalmente a una popolazione dal basso capitale culturale, oltre che economico (nella provincia il 51\% della popolazione non ha alcun titolo di studio oppure ha una istruzione di base. Istat 2021), De Luca si mostra abilissimo e "demografico" nel rappresentare gli interessi del suo ceto di riferimento. Secondo un canone revanchista consolidatissimo, in questa città divenuta marginale, l'uomo politico incarna la nostalgia di una grandezza svanita, la messa al bando dei responsabili e la promessa di un ritorno agli antichi fasti. Insieme alla pubblicità di negozi, ristoranti e attività varie, che simboleggiano l'impresa e la voglia di fare, e i cui marchi vengono esibiti nelle dirette del primo cittadino congiuntamente ai prodotti (cibo, camicie etc. vengono infatti regolarmente mostrati in video), quella del sindaco è soprattutto la rappresentazione della lotta contro le inciviltà e i nemici interni alla comunità: le élite che l'hanno ridotta allo stato attuale, e gli incivili che la lordano e che hanno reso ordinaria e visibile l'illegalità. Questi ultimi sono frequentemente semplici cittadini, ma più spesso traslocatori o rigattieri improvvisati, che si sbarazzano come possono del loro carico, creando discariche. Ma sono soprattutto gli ambulanti che, in linea del resto con altri populismi contigui meridionali (si veda il caso di Salerno, esplorato da Avallone e Nïang 2021), diventano l'oggetto di un esercizio capriccioso e ostentato del potere, che prima li costringe a emergere e poi li vessa con sequestri, con sospensioni arbitrarie delle licenze e con richieste sempre nuove e dispendiose di produrre nuovi documenti e adeguamenti. Per esempio con l'imposizione di cambiare il colore dei tendaggi che coprono gli stand delle rivendite di frutta e verdura; o, magari, con quella di aumentare costantemente la distanza tra un banchetto e l'altro, tra un camion e l'altro, costringendo così gli operatori a adattamenti logistici sempre nuovi e, soprattutto, erodendo gradualmente quella convenienza economica che, nel caso del commercio ambulante, presiede di norma alla scelta dei luoghi intorno ai quali insediarsi. $\mathrm{O}$, ancora, con l'interdizione ai venditori ambulanti di generi alimentari di esercitare la propria attività in prossimità di quelle arterie rivierasche che, in una città di mare come Messina, costituiscono nella stagione estiva il vero centro della vita sociale ed economica. Il tutto a beneficio di quegli esercenti di lidi e di negozi che costituiscono, per "qualità" e peso fiscale gli interlocutori privilegiati dell'azione politica locale (è infatti la dimensione dell'attività, che nel caso di un negozio sarà quasi sempre superiore a quella di un camion o di una moto-ape, a determinare la tassazione e la convenienza istituzionale a coltivare una particolare popolazione. Senza contare il modo in cui le attività commerciali si connettono a un progetto più generale di città. Per esempio la città turistica).

Qualcosa, peraltro, che non appare slegata dall'accostamento, piuttosto comune nelle rappresentazioni di senso comune e giornalistico, tra vendita ambulante, inciviltà, violenza e criminalità. Tale considerazione appare come una conseguenza del fatto che, alla stregua dell'edilizia, l'ambulantato è un settore che impiega individui con scarsa o nulla qualificazione, frequentemente appartenenti al sottoproletariato urbano. Ossia ciò che - non diversamente da quanto si riscontra in altre città meridionali alle prese con conflitti culturali e di classe di lunga durata (Petrillo 2011; Pine 2015) - agli occhi degli individui e dei ceti maggiormente integrati incarna una fastidiosa forma di alte- 
rità interna alla società locale, per via degli atteggiamenti pubblici, dell'aspetto, della musica ascoltata e di vari altri elementi riconosciuti dall'intimità culturale, che fanno convenire la maggioranza «distinta» su una percezione di questa classe «bassa» come culturalmente arretrata e minacciosa. Inoltre coloro che optano per la vendita ambulante presentano frequentemente precedenti penali che, ai sensi della normativa vigente, li escludono a lungo dalla facoltà di ottenere licenze commerciali. Per questo stesso motivo molti di loro - decisi a uscire in parte o del tutto da traiettorie biografiche autenticamente criminali - non possono fare altro che esercitare irregolarmente la propria attività commerciale di merci lecite (oppure ricorrere a prestanomi, da rinvenire in ambito familiare o esterno, procedendo magari ad arrangiamenti di varia natura suscettibili in alcuni casi di generare vari problemi con partner, fornitori, polizia etc.). Una condizione di irregolarità che storicamente - ben prima cioè delle campagne "desoteane" per l'emersione perseguite dalla giunta De Luca - li espone a tensioni con le forze dell'ordine e a saltuari scontri con l'annona che, almeno in un paio di occasioni, hanno assunto la forma di vere e proprie rivolte urbane. L'animosità e l'afflittività esplicita e manifesta di cui questa particolare popolazione di commercianti è destinataria, ha dunque ragioni strutturali e sovrastrutturali di lunga durata. Nella prospettiva di De Luca l'emersione forzata di tale categoria di commercianti - perseguita attraverso una serie infinita di sequestri da parte del locale corpo di vigili urbani - è tanto un contributo a una nozione generale di legalità quanto una sorta di regolamento di conti tra l'istituzione e questo ceto sociale «riottoso», non semplicemente popolare ma autenticamente «plebeo»: ossia pressoché "naturalmente" refrattario alla disciplina e alle regole. L'esatto contrario di quanto previsto dal mito e dal racconto deluchiano di sé stesso, che delle origini umili e contadine così come dell'immagine dell'uomo povero che si costruisce con i propri soli mezzi, parte dal paesello natio con le valige di cartone e costruisce una fortuna attraverso una dura dedizione ai fini, fa da sempre il proprio punto di forza (si vedano a riguardo le dichiarazioni raccolte da De Angeli 2021). Una narrazione di sé, ma anche un ricorso a consolidati miti pubblici non solo nazionali sulla figura dell'imprenditore (nel caso italiano già visto in azione con Berlusconi) che agisce da riflesso per tutti coloro che, come i commercianti legittimi, possono riconoscersi nell'etica deluchiana del lavoro e in una conseguente pretesa ai privilegi. Qualcosa del resto che è parte del senso comune generale, ben oltre il «mondo dell'impresa», e che fonda le proprie ragioni sul rifiuto o la minimizzazione del ruolo delle strutture sociali nel determinare le traiettorie individuali (in primis l'istituzione giudiziaria, che, una volta catturate le persone, preclude a molte di loro la possibilità di un autentico recupero) e sul mancato riconoscimento degli effetti della diseguale distribuzione delle opportunità (lì ove, anche ai "piani più bassi" del sociale, una apparentemente minima differenza nei redditi o nei livelli di istruzione familiare può effettivamente produrre effetti apprezzabili relativamente al rapporto degli individui con la formazione, il lavoro e la progettualità biografica. Si veda a riguardo: Baglioni 2021: 38 passim).

In modo analogo ad altre esperienze politiche contemporanee, quello di De Luca appare perciò come un progetto populista che, di fronte all'informalizzazione de facto di una città - in cui i bassissimi salari hanno costretto decine di migliaia di persone a emigrare o ad arrangiarsi, anche quando dispongono di una pensione o di un reddito regolare - seleziona le forme "degne" di cittadinanza (ossia ancora munite di interessi da tutelare, quali un immobile da far fruttare) e dà loro orgoglio, dividendo così artificialmente una società ormai generalmente impoverita. Eliminare la concorrenza degli ambulanti regolari e irregolari significa infatti garantirsi la fedeltà della vasta platea della piccola-borghesia del commercio e di quella proprietaria delle botteghe, che dai commercianti traggono i fitti; con il vantaggio aggiunto, inoltre, di garantire le entrate fiscali necessarie alle casse comunali. Un modo di andare alle briciole, garantendosi una reputazione e un futuro politico fondato sull'efficienza, che ha la presidenza della Regione come traguardo dichiarato.

\section{CONCLUSIONI}

Alla luce di quanto sin qui osservato, l'economia informale appare come il cuscinetto di quelle periodiche ristrutturazioni che garantiscono, tanto al capitale nel proprio complesso quanto alla società e alla politica, di potere tollerare il costo delle transizioni e delle riorganizzazioni periodiche dei modi di produzione e ridistribuzione della ricchezza (secondo modalità per lo più orizzontali, ossia interne alle classi superiori). L'impossibilità di una via 
informale di risoluzione delle tensioni segnerebbe infatti la dissoluzione del sociale e costituirebbe il preludio per insorgenze scomposte. L'informalità appare dunque come ciò che è essenziale alla riproduzione della vita e dell'ordine politico sotto il capitalismo e dentro l'organizzazione statale come la conosciamo.

Al contempo l'informalità costituisce uno strumento simbolico potentissimo, utile a quelle mobilitazioni culturali o sovrastrutturali che stanno alla base delle gerarchie e delle alleanze tra settori o classi sociali. L'informale è tutto quel che sfugge alle definizioni ed è dunque fluido, finendo con l'includere ciò che è apparentemente opposto, tanto sul piano materiale quanto simbolico: i ricchi, i poveri, i benestanti decaduti etc. È, inoltre una condizione che riguarda molti, sia pure con gradualità diverse. E che per questo costituisce una sorta di surplus, insieme simbolico e materiale, a cui poteri politici raffinati - o finto grossolani - possono fare appello per mobilitare sezioni della popolazione contro le altre, e assicurarsi quote essenziali di consenso. Non è dunque un caso che - nella cornice di quella che potremmo chiamare la condizione fisiologica e strutturale del clientelismo, fondamentali per la vita di democrazie che, come quelle contemporanee, sono basate sulle alleanze - gli spazi "informali" sono stati spesso riprodotti e curati dal mondo politico al fine di gestire i rapporti dei gruppi marginali con le istituzioni formali.

Ciò che da tutto questo deriva ai fini scientifici più che politici (la politica ne deve essere infatti già consapevole) è che l'informale non andrebbe mai rappresentato come un alter rispetto ai mondi ufficiali, e che qualunque definizione realista dei rapporti tra i due non dovrebbe tanto sottolineare il continuum tra i settori, quanto la loro sostanziale identità e sovrapponibilità. È questo, del resto, ciò che di fatto già accade - per l'appunto informalmente; sul piano di una "realpolitik" istituzionale - e che ha reso possibili riforme del lavoro e del welfare altrimenti semplicemente impraticabili.

\section{RIFERIMENTI BIBLIOGRAFICI}

Abu-Lughod L. (1990), The Romance of Resistance: Tracing Transformations of Power through Bedouin Women, in «American Ethnologist», 17, 1: 41-55.

Alden J. (1981), Holding Two Jobs: An Examination of Moonlighting, in S. Henry, Informal Institutions: Alternative Networks in the Corporate State, New York: St. Martin's Press.

Allum P. (1975), Potere e società a Napoli nel dopoguerra, Torino: Einaudi.

Althusser L. (1976), Sull'ideologia, Bari: Dedalo.

Anselmi, M. (2017), Populismo. Teorie e problemi, Milano: Mondadori.

Avallone G., Niang D. (2020, cur.), Vivere non è un reato. Lavoro ambulante e diritto alla città, Verona: Ombre Corte.

Baglioni, G. (2021), Benessere e fragilità: La mobilità sociale in Italia, Milano: Franco Angeli.

Biancalana C. (2020), Four Italian Populisms, in P. Blokker e M. Anselmi, Multiple Populisms. Italy as Democracy's Mirror, London-New York: Routledge.

Blokker P., Anselmi M. (2020, eds.), Multiple Populisms. Italy as Democracy's Mirror, London/New York: Routledge

Azzarà S.G. (2018), Comunisti, fascisti e questione nazionale. Fronte rossobruno o guerra d'egemonia?, Milano: Mimesis.

Berger M., Buvinic M. (1989), Women's Ventures: Assistance to the Informal Sector in Latin America, Boulder: Kumarian Press.

Binik O. (2017), Quando il crimine è sublime: La fascinazione per la violenza nella società contemporanea, Milano: Mimesis.

Bolzoni M., Gargiulo E., Manocchi M. (2015), The Social Consequences of the Denied Access to Housing for Refugees in Urban Settings: The Case of Turin, Italy, in «International Journal of Housing Policy»,15, 4: 400-417.

Bourdieu P. (1993, dir.) La Misère du monde, Paris: Éditions du Seuil.

Bowe J., Bowe M., Streeter S. (2000), Gig. Americans Talk About their Jobs, New York: Three Rivers Press.

Brighenti, M.A. (2010), Visibility in Social Theory and Social Research, New York: Palgrave MacMillan. 
Broad D. (2000), The Periodic Casualization of Work: The Informal Economy, Casual Labor and the Long Durée, in F. Tabak, M.A. Crichlow, Informalization. Process and Structure, Baltimore/London: The John Hopkins University Press.

Canovan M. (1981), Populism, New York: Harcourt Brace Jovanovich.

Carlini R. (2019), L'Italia nella bolla del lavoro precario, in «Internazionale», 21 febbraio, https://www.internazionale.it/opinione/roberta-carlini/2019/02/21/dati-industria-lavoro

Chubb, J. (1983), Patronage, Power, and Poverty in Southern Italy. A Tale of Two Cities, Cambridge: Cambridge University Press.

Coletto D. (2010), The Informal Economy and Employment in Brazil: Latin America, Modernization, and Social Changes, New York: Palgrave MacMillan.

Crainz G. (2005), Storia del miracolo italiano. Culture, identità, trasformazioni fra anni cinquanta e sessanta, Roma: Donzelli.

Crainz G. (2012), Il paese reale: dall'assassinio di Moro all'Italia di oggi, Roma: Donzelli.

Crouch C. (2019), Se il lavoro si fa gig, Bologna: il Mulino.

De Angeli A. (2021), Cateno De Luca dopo il concerto alla villa Dante: "Il mio modesto contributo per dare una svolta artistica e sociale alla nostra città" in, «Messina7.it», 7 settembre, https://messina7.it/2021/09/07/catenode-luca-dopo-il-concerto-alla-villa-dante-il-mio-modesto-contributo-per-dare-una-svolta-artistica-e-sociale-allanostra-citta/

De Federicis N. (2017), Populismo, plebiscitarismo e crisi della democrazia, in «Teoria politica» 7: 155-179.

De Genova N. (2002), Migrant "Illegality" and Deportability in Everyday Life, in «Annual Review of Anthropo$\log \gg$, , 31: 419-447.

De Soto H. (2000), The Mystery of Capital: Why Capitalism Triumphs in the West and Fails Everywhere Else, New York: Basic Books.

Doeringer P, Piore M. (1971), Internal Labor Markets and Manpower Analysis, Lexington: Heath/Lexington Books.

Durkheim, E. (1893), La divisione del lavoro sociale, Edizioni di Comunità: Milano, 1962.

Esposito M. (2012), Il contrasto al lavoro nero: discontinuità dei percorsi legislativi e cultura dei valori giuridici, Napoli: Discussion Paper Series, Crisei.

Esposito E., Chiodelli F. (2020), Occupazioni abusive in un contesto di edilizia residenziale pubblica a Napoli: una pratica individualistica?, in «Argomenti», 15: 59-76.

Evans-Pritchard E.E. (1940), The Nuer: A Description of the Modes of Livelihood and Political Institutions of a Nilotic People, Oxford: Clarendon Press.

Fantozzi P., Raniolo F (2018), Clientelismo, privatizzazione del pubblico e governo di partito, in «Quaderni di Sociologia $\gg, 78:$ 11-39.

Farinella D., Saitta P. (2019), The Endless Reconstruction and Modern Disasters: The Management of Urban Space Through an Earthquake - Messina, 1908-2018, Cham: Palgrave MacMillan.

Farmer P. (2004), An Anthropology of Structural Violence, in «Current Anthropology», 45, 3: 305-325.

Ferman L., Henry S., Hoyman M. (1987), Issues and Prospects for the Study of Informal Economies: Concepts, Research Strategies and Policy, in «The Annals of American Academy of Political Science», 493: 154-72.

Ferrera M. (2006), Le politiche sociali: l'Italia in prospettiva comparata, Bologna: il Mulino.

Fisher M. (2009), Capitalist Realism: Is There No Alternative?, London: Zero Books.

Fregolent L., Torri, R. (2018, cur.), L'Italia senza casa. Bisogni emergenti e politiche per l'abitare, Milano: FrancoAngeli.

Gallino L. (1975), Politica dell'occupazione e seconda professione, in «Economia e lavoro», IX, 1: 81-95.

Germani, G. (1978), Authoritarianism, Fascism, and National Populism, New Brunswick: Transaction Book.

Giannini M. (2017), Precarietà, una categoria ambigua. Radici sociali e percezioni differenti nei contesti socio-economici, in P. Bouffartigue, M. Giannini e A. Lamanthe, Travail et crise: l'Europe du Sud, un laboratoire?, Milano: FrancoAngeli. 
Ginsborg, P. (1989), Storia d'Italia dal dopoguerra a oggi, Torino: Einaudi.

Gutmann P. M. (1978), Are the Unemployed, Unemployed?, in «Financial Analysts Journal», 34, 5: 26-29.

Hart K. (1973), Informal Income Opportunities and Urban Employment in Ghana, in «Journal of Modern African Studies», 11, 1: 61-89.

Holston J. (2009), Insurgent Citizenship: Disjunctions of Democracy and Modernity in Brazil, Princeton: Princeton University Press.

Istat (2021), Il censimento permanente della popolazione in Sicilia. Prima diffusione dei dati definitivi 2018 e 2019, Roma: Istat.

Jensen L. (2016, ed.), The Roots of Nationalism. National Identity Formation in Early Modern Europe, 1600-1815, Amsterdam: Amsterdam University Press.

Jesi F. (1979), Cultura di destra, Milano: Garzanti.

Laclau E. (2008), La ragione populista, Roma/Bari: Laterza.

Limosani M. (2021), Messina: un'istantanea sull'economia della città, Messina: Università di Messina - Parliament Watch.

Losby J.L., Else, J.F., Kingslow, M.E., Edgcomb, E.L., Malm, E.T., Kao, V. (2002), Informal Economy Literature Review, Washington/Newark: The Aspen Institute-Ised.

Memmi, A. (1979) Ritratto del colonizzato e del colonizzatore, Napoli: Liguori.

Mezzadra S., Neilson B. (2013), Border as Method, or, the Multiplication of Labour, Durham: Duke University Press.

Mukhija V., Loukaitou-Sideris A. (2014), Introduction, in V. Mukhija e A. Loukaitou-Sideris, The Informal American City. Beyond Taco Trucks and Day Labor, Cambridge/London: Mit press.

Murgia A. (2010), Dalla precarietà lavorativa alla precarietà sociale. Biografie in transito tra lavoro e non lavoro, Città di Castello (PG): I libri di Emil.

Hansen K.T., Vaa M. (2004), Introduction, in K.T. Hansen e M. Vaa, Reconsidering Informality. Perspectives from Urban Africa, Uppsala: Nordiska Afrikainstitutet.

Ortner, Sherry (1995), Resistance and the Problem of Ethnographic Refusal, in «Comparative Studies in Society and History», 37: 173-193.

Panarari M. (2010), L'egemonia sottoculturale. L'italia da Gramsci al gossip, Torino: Einaudi.

Petrillo, A. (2011), Napoli globale: discorsi, territorio e potere nella "città plebea", in S. Palidda, La rivoluzione liberista nelle città euro-mediterranee, Messina: Mesogea.

Pine J. (2015), Napoli sotto traccia. Camorra, «zona grigia» e arte di arrangiarsi. Musica neomelodica e marginalità sociale, Roma: Donzelli.

Pozzi, G. (2020), Fuori casa. Antropologia degli sfratti a Milano, Milano: Ledizioni.

Shapland J. (2009), Potential Effects of National Policies on the Informal economy, in J. Shapland e P. Ponsaers, The Informal economy and Connections with Organised Crime: The Impact of National Social and Economic Policies, Den Haag: Boom Juridische Uitgevers.

Raijman R. (2001), Mexican Immigrants and Informal Self-Employment in Chicago, in «Human Organization», 60, $1: 47-55$.

Harris R. (2007), The Assumptionists and the Dreyfus Affair, in «Past \& Present», 194,1: 175-211.

Saitta P. (2020), Fenomenologia di un neo-populista. Declinazioni urbane dell'autoritarismo: il caso di Messina, in «Argomenti», 15: 123-150.

Sanò G. (2018), Spazio prodotto e Spazio produttore. La relazione tra il sistema di accoglienza e due territori a Messina, in M. Bergamaschi e V. Piro, Sociologia urbana e rurale, Milano: FrancoAngeli.

Sassen, S. (1994), Cities in a World Economy, Thousand Oaks: Pine Forge Press.

Scott, J.C. (1990), Domination and the Arts of Resistance: Hidden Transcripts, New Haven: Yale University Press.

Scott J.C. (1998), Seeing Like a State: How Certain Schemes to Improve the Human Condition Have Failed, New Haven: Yale University Press.

Stanley B. (2008), The Thin Ideology of Populism, in «Journal of Political Ideologies, 13, 1: 95-110. 
Tassinari A., Maccarrone, V. (2018), Le mobilitazioni dei fattorini della gig economy in Italia: forme organizzative e implicazioni per la rappresentanza, in «Quaderni di Rassegna Sindacale: Lavori», 2: 75-106.

Toffanin, T. (2016), Fabbriche invisibili. Storie di donne, lavoranti a domicilio, Verona: Ombre Corte.

Trevor-Roper H. (1969), Protestantesimo e trasformazione sociale, Roma/Bari: Laterza.

Ventura R.A. (2017), Teoria della classe disagiata, Roma: Minimum Fax.

Wallerstein I. (1983), Historical Capitalism, London: Verso. 